\title{
El papel del trabajo interdisciplinario entre Psicología y Derecho en el abordaje a la violencia ejercida en relaciones de pareja: un análisis a partir de un proceso de intervención ${ }^{1}$
}

\author{
The role of interdisciplinary work between psychology and law in \\ addressing violence in intimate partner relationships: an analysis \\ based on an intervention process
}

Marta María Artavia Alpízar ${ }^{2}$

\begin{abstract}
RESUMEN
Este artículo analiza la importancia del trabajo interdisciplinario en el abordaje de la violencia ejercida en relaciones de pareja, por medio del análisis de una experiencia realizada bajo la modalidad de Práctica Dirigida para optar por el grado de Licenciatura en Psicología, en el Consultorio Jurídico de la Universidad de Costa Rica, Defensoría de los Habitantes. A partir de los resultados obtenidos, se logra identificar que los factores de riesgo de femicidio en las 13 mujeres atendidas disminuyó gracias al trabajo interdisciplinario entre Psicología y Derecho. De esta manera, se concluye que para el abordaje a la violencia es necesario trabajar de forma interdisciplinaria para así construir espacios más seguros de atención y estrategias que permitan reducir el riesgo de femicidio.
\end{abstract}

Palabras clave: Violencia Ejercida en Relaciones de Pareja, ruta crítica, acompañamiento psicológico, intervención en crisis, interdisciplinariedad.

\footnotetext{
${ }^{1}$ Este trabajo es un análisis que parte del Trabajo Final de Graduación realizado para optar por el grado de licenciatura en Psicología, en donde se contó con la revisión de la Licda. Paola Brenes (Directora), la M.Sc. Adriana Rodríguez Fernández (Lectora), el Lic. Ruthman Moreira Chavarría (Lectora), la M.Sc. Teresita Ramellini Centella (Directora del Tribunal) y la Licda. Nioe Víquez Moreno (Profesora Invitada).

${ }^{2}$ Universidad de Costa Rica. San José, Costa Rica. Correo electrónico: martaartavia@gmail.com ORCID: https://orcid.org/0000-0001-6100-7183
}

DOI: 10.15517/WL.V16I2.48417

Recepción: 20/02/2021 Aceptación: 11/08/2021 


\begin{abstract}
This article analyzes the importance of interdisciplinary work in addressing violence in intimate partner relationships, through the analysis of an experience carried out under the modality of Directed Practice to obtain a degree in Psychology, at the Consultorio Jurídico de la Universidad de Costa Rica, Defensoría de los Habitantes. From the results obtained, it is possible to identify that the risk factors of femicide to the 13 women attended decreased thanks to the interdisciplinary work between Psychology and Law. Thus, it is concluded that in order to address violence it is necessary to work in an interdisciplinary manner in order to build safer spaces for care and strategies to reduce the risk of femicide.
\end{abstract}

Key Words: Violence in relationships, critical route, psychological support, crisis intervention, interdisciplinary approach.

\title{
Introducción
}

La violencia contra la mujer (VCM), según la Organización de Estados Americanos (OEA), a través de la Convención Interamericana para Prevenir, Sancionar y Erradicar la Violencia Contra la Mujer (Convención Belém Do Pará), de 1994, es definida como cualquier conducta que busca lastimar y/o asesinar a las mujeres debido a su condición de género. Asimismo, cualquiera de sus manifestaciones físicas, sexuales y/o psicológicas, pueden originarse en distintos contextos como lo son relaciones de pareja, a nivel comunitario, en espacios laborales o inclusive al solicitar servicios de atención en instituciones, lo que afecta a múltiples poblaciones (OEA 1994).

De esta manera, según la Organización Mundial de la Salud (OMS) la VCM es un problema social que afecta la salud a nivel público, ya que es una violación a los derechos humanos, lo que significa que las condiciones de vida se ven afectadas al no contar con las mismas oportunidades y disfrute de sus derechos (OMS 2017).

Respecto a consecuencias físicas y psicológicas, Walker (2009) y Mahapatro (2018) determinan que, por la dinámica de la violencia, su intensidad y constancia puede deteriorar a corto y largo plazo la salud reproductiva de las mujeres, pero también generar otras lesiones físicas severas. Asimismo, desde el punto de vista psicológico, el control, los celos, humillaciones y otras formas de violencia pueden generar miedo y estrés de forma prolongada, lo que genera a largo plazo condiciones de salud como dolor de cabeza crónico, hipertensión y otras. 


\section{ranum}

Wimblu, Rev. Estud. de Psicología UCR, 16(2) 2021 (Julio-Diciembre): 119-136 /ISSN: 1659-2107

Por su parte, desde un punto de vista más estadístico, en el caso de la Violencia Ejercida en Relaciones de Pareja (VERP), a nivel mundial la OMS (2017) menciona que 1 de cada 3 mujeres fueron víctimas, lo que equivale a un 35\% de la población. Además, un $38 \%$ de los femicidios fueron ejecutados por parte de parejas violentas.

Aunado a lo anterior, en Costa Rica, la Encuesta Nacional de Violencia contra las Mujeres en el año 2003, logró identificar que, desde los 16 años, el 45.4\% de las mujeres experimentaron violencia física, un $46.6 \%$ psicológica y un 78\% sexual (Sagot y Guzmán 2004). Igualmente, el Observatorio de Violencia de Género contra las Mujeres y el Acceso a la Justicia (2020), registra que entre el 2010 y el 2019 se solicitaron 133 medidas de protección diarias, para un total aproximado de 485 276. En cuanto a los roles en la dinámica de violencia, 51187 (79\%) de las personas agresoras fueron hombres y 52534 (80\%) de las víctimas fueron mujeres.

Respecto a los femicidios, en el 2017 se contabilizaron 26 casos, en el 2018 hubo un total de 24, en el 2019 se reportaron 15 y al 16 de diciembre del 2020, 11 mujeres fueron asesinadas por su pareja (Observatorio de Violencia de Género contra las Mujeres y Acceso a la Justicia 2020).

A partir de dicha problemática, se ha buscado crear procesos conjuntos entre Psicología y Derecho que generen factores protectores para disminuir el riesgo de femicidio, por medio de estrategias como la intervención en crisis, la creación de planes de seguridad y los distintos mecanismos legales para promover el respeto de los derechos humanos y el acceso a la justicia pronta y cumplida (Cervantes y Veraza 2018).

Es por lo anterior, que se considera vital reflexionar sobre la importancia del trabajo interdisciplinario, entre la carrera de Psicología y Derecho, en el abordaje a la VERP, para generar espacios de intervención que permitan humanizar la ruta crítica y prevenir el riego de femicidio. Para propiciar este análisis, el presente documento mencionará las características de la VERP, la ruta crítica, el acompañamiento psicológico e intervención en crisis al abordar mujeres víctimas de violencia, pero también se hará una comparación de estos elementos, por medio de la descripción y análisis de una experiencia desarrollada como práctica dirigida para optar por el grado de Licenciatura en Psicología, de la Universidad de Costa Rica, realizada entre el 2019 y el 2020, con el fin de fortalecer el servicio por el 
Consultorio Jurídico de la Universidad de Costa Rica, con sede en la Defensoría de los Habitantes.

\section{Características de la VERP, su rol en el sistema patriarcal y sus consecuencias}

Castro (2015) menciona que la VERP es toda conducta que se realiza en el contexto de relación de pareja, que se ejecuta para afectar o propiciar la pérdida de la integridad física, sexual, psicológica o patrimonial de una persona, para controlarla y dominarla. Según el estudio de Castro (2015) y Sagot, Carcedo y Guido (2000), generalmente, la persona agresora es un hombre y las víctimas son mujeres y niñas.

Aunado a lo anterior, el poder es utilizado para generar conductas violentas progresivamente, por medio del aislamiento de redes de apoyo, la utilización de amenazas y el control sobre las decisiones, lo que violentan los derechos humanos, al limitar su capacidad de disfrutarlos, lo cual genera en las mujeres miedo constante, al no tener certeza de cuándo puede ocurrir el siguiente episodio de violencia (Abrahams 2010).

Desde el punto de vista sociopolítico, la VERP perpetúa el sistema patriarcal al fundamentar las agresiones y la violencia en el conservadurismo político y religioso, que promueven condiciones de desigualdad, donde las mujeres son consideradas como inferiores y los hombres superiores (Instituto Nacional de las Mujeres 2016). Esta condición puede agraviarse al no contar con las mismas oportunidades laborales que los hombres, lo que propicia la dependencia económica para subsistir (Abrahams 2010). De esta manera, las mujeres deben cumplir con su rol de madres y esposas, sin importar el nivel de riesgo al tener una relación amorosa con un compañero que no posee empatía, que ejerce conductas agresivas y que la visualiza como un objeto de su pertenencia (Ulate 2008).

Es a partir de las dinámicas anteriores que la European Union Agency for Fundamental Rights (FRA) establece que la violencia, a nivel psicológico, se da por medio de la intimidación, el control de las decisiones, el chantaje y el aislamiento (Castro 2015; FRA 2015). Además, pueden utilizarse golpes, pellizcos y armas para afectar la integridad física. En el caso de la violencia sexual, se obliga a las mujeres a realizar prácticas sexuales 
contra su voluntad; y a partir de la violencia patrimonial se atenta contra los bienes materiales que son necesarios para subsistir (Castro, 2015).

Debido a las distintas formas de violencia, a nivel físico, las mujeres pueden sufrir abortos, problemas de infertilidad, desnutrición, enfermedades de transmisión sexual, entre otras condiciones. Psicológicamente, el miedo y los altos niveles de estrés propician el desarrollo de depresión, ideaciones suicidas, trastornos de estrés postraumático (TEP) y desesperanza aprendida (Mahapatro 2018; Walker 2009).

De esta manera, se visualiza que las consecuencias generadas por la violencia y la promoción de situaciones de desigualdad entre hombres y mujeres, puede afectar la calidad de vida de las mujeres (Abrahams 2010; Castro 2015; FRA 2015; Instituto Nacional de las Mujeres 2016; Ulate 2008).

\section{Ruta crítica}

Jiménez, Blanco, Medina y Gómez (2013) establecen que la ruta crítica es el proceso en que una víctima de VERP decide actuar para protegerse de la violencia y sus consecuencias.

Aunado a lo anterior, las decisiones de las víctimas pueden cambiar, ya que al solicitar ayuda surgen contradicciones y momentos críticos, como, por ejemplo: sentir miedo y culpa al comunicar que son víctimas, lo que hace que puedan retractarse de sus decisiones. Además, si su compañero se entera de la situación, podría aumentar la violencia, lo que la expone a situaciones de riesgo y necesite solicitar medidas de protección.

\section{Acompañamiento psicológico en el abordaje de la VERP}

Raffo (2007), menciona que, el acompañamiento psicológico a mujeres afectadas por la violencia se caracteriza por ser un proceso en el que se brinda contención emocional, durante procesos legales, lo que permite prevenir la revictimización, pero también dar un 
espacio al dolor, por medio de la escucha activa y atenta, el respeto al silencio y la validación emocional, lo que promueve el fortalecimiento de las usuarias al reafirmarse su valor como persona y el respeto por sus derechos.

Aunado a lo anterior, de forma integral, las personas profesionales en psicología pueden acompañar al personal de Derecho a audiencias judiciales, con el fin de promover la validación de las emociones de las usuarias y apoyar el manejo de situaciones de crisis, antes y después de los procesos legales (Raffo 2007).

Desde el punto de vista de servicios atencionales, se puede dar información a las víctimas sobre distintos mecanismos institucionales a los que se puede tener acceso para protegerse de la violencia, a partir de las características de los procesos legales (Raffo 2007).

Finalmente, el acompañamiento psicológico no se limita solamente a dar procesos de contención emocional a las usuarias, sino que se puede brindar apoyo a las personas profesionales de Derecho, para así construir herramientas de forma conjunta para proteger a la usuaria y promover el respeto de sus derechos humanos (Raffo 2007).

\section{Intervención en crisis para la atención de mujeres afectadas por VERP}

Roberts (2005) define la intervención en crisis como un período de inestabilidad psicológica, que se genera ante la exposición a eventos considerados como peligrosos, ya que las personas no pueden solucionar sus problemas con los recursos personales que normalmente utilizan, por lo que surge el miedo y la ansiedad. A pesar de esto, la crisis representa una oportunidad para generar cambios positivos, si se logran adquirir nuevas herramientas y habilidades (Roberts 2005; Yeager y Roberts 2015). De esta manera, la intervención en crisis busca recuperar ese equilibro al contar con nuevas estrategias para afrontar las situaciones (Yeager y Roberts 2015).

En el caso específico de las mujeres víctimas de VERP, Claramunt (1999) y Roberts (2005) mencionan que, el principal objetivo de la intervención en crisis es crear espacios seguros de intervención, para propiciar la reducción de factores de riesgo ante el femicidio. Para ello, se busca fortalecer las redes de apoyo y la autoconfianza, por medio de estrategias 
como planes de seguridad, que permitan la protección y atención a las necesidades de las mujeres en riesgo, a través del contacto con instituciones.

Finalmente, durante el proceso de atención Claramunt (1999) recomienda brindar apoyo emocional, escucha atenta, realizar preguntas abiertas, analizar recursos personales y redes de apoyo que podrían atender a las usuarias en casos de emergencia. Así, al tomar en cuenta la crisis, sus características y consecuencias, se crea todo un plan de acción que permite enfrentar situaciones de riesgo.

\section{Descripción de la experiencia de Trabajo Final de Graduación y sus principales hallazgos}

Para optar al título de Licenciatura en Psicología de la Universidad de Costa Rica, se realizó una Práctica Dirigida en las instalaciones del Consultorio Jurídico de la Universidad de Costa Rica, con sede en la Defensoría de los Habitantes, durante un total de 7 meses a tiempo completo. Durante esta experiencia, se buscó fortalecer el servicio de atención brindado a usuarias afectadas por VERP, desde el enfoque género sensitivo y de derechos humanos, lo que permitió a las usuarias mejorar sus condiciones de vida (Artavia 2021). Por este motivo, a continuación, se presentan las características del sistema de Consultorios Jurídicos, así como datos relevantes sobre el Consultorio Jurídico con sede en la Defensoría de los Habitantes (CJDHR) en relación con la VERP y el rol de la Psicología en la institución. Posterior a esto, se presenta la metodología, los principales resultados y conclusiones obtenidos a partir de la intervención en las usuarias.

\section{Origen de los Consultorios Jurídicos de la Universidad de Costa Rica}

En 1967 se crea el sistema de Consultorios Jurídicos de la Universidad de Costa Rica, con el fin de brindar servicios de acompañamiento legal a personas en condiciones de vulnerabilidad, a las que se les dificultaba contratar servicios profesionales. Asimismo, se buscó que las personas estudiantes tuvieran la oportunidad de mejorar su formación, al 
conocer mejor las realidades socioeconómicas de sus clientes (Alvarado, Esquivel y Álvarez 1982; Rodríguez 2001). Es importante destacar que, en 1971 se reconoce la figura legal de los Consultorios Jurídicos de la Universidad de Costa Rica, a través de la Ley $\mathrm{N}^{\circ} 4775$, denominada Ley de Consultorios y se da amparo a aquellas personas en condición de vulnerabilidad, a través de asesoramiento legal gratuito. Además, se autoriza a las personas estudiantes de Derecho a acompañar a las personas usuarias en procesos de audiencia y trámites legales (Facultad de Derecho 2014; Ley de Consultorios 1971).

En el caso del CJDHR, esta entidad surge tras la creación de la Defensoría de los Habitantes en 1980, al establecerse el acuerdo con la Universidad de Costa Rica, la Defensoría de los Habitentes se compromete a brindar los medios económicos para su accionar (Defensoría de los Habitantes 2010).

Finalmente, para el 2014 se reportó un total de 22 Consultorios Jurídicos a los largo del país para brindarle atención a personas en condición de vulnerabilidad, por medio del trabajo de personas estudiantes de derecho, a través de sus 208 horas de trabajo en temáticas sobre derecho familiar, laboral, conflictos por violencia doméstica, entre otros (Facultad de Derecho 2014; Mónica Sancho, comunicación personal, 1 de diciembre del 2017; Rodríguez 2001).

\section{Datos relevantes sobre el CJDHR con relación a la VERP}

A nivel estadístico, el CJDHR reporta que durante el 2018 tuvieron 300 casos activos, pero que 75 fueron víctimas de VERP (Mónica Sancho, comunicación personal, 26 de marzo del 2019). Además, al 4 de febrero del año 2020 la institución contó con 286 procesos activos, de los cuales, 133 fueron mujeres afectadas por consecuencias generadas a partir de la VERP (Mónica Sancho, comunicación personal, 4 de febrero del 2020).

2 De esta manera, los datos reflejan que desde el 2018 al 2019 se dio un incremento de 58 víctimas de violencia, lo que muestra que la mayoría de los procesos de la institución se se relacionan con mujeres que son agredidas por sus parejas o familias (Mónica Sancho, comunicación personal, 4 de febrero del 2020) 


\section{Rol de la Psicología en el trabajo interdisciplinario y el CJDHR}

Los servicios ofrecidos por el CJDHR se realizan por medio del trabajo interdisciplinario entre las carreras de Psicología y Derecho. A nivel de Psicología, estudiantes del Módulo Optativo de Psicología y Violencia Doméstica, han brindado acompañamiento psicológico e intervención en crisis a mujeres afectadas por VERP, a partir de enfoques de derechos humanos y género sensitivos (Mónica Sancho, comunicación personal, 26 de marzo del 2019).

Aunado a lo anterior, el estudiantado de Derecho y Psicología ha adquirido herramientas para trabajar de forma interdisciplinaria, especialmente, al valorar el riesgo de femicidio al que pueden exponerse las personas usuarias. Una vez identificadas las conductas violentas ejecutadas por la persona agresora, desde una perspectiva legal, se ha dado asesoría sobre medidas de protección y se les motivó a utilizar siempre la empatía como recurso para promover la expresión emocional y la toma de decisiones (Mónica Sancho, comunicación personal, 26 de marzo del 2019).

Finalmente, a nivel de resultados, la Directora del CJDHR, la Lic. Mónica Sancho comenta que el apoyo de Psicología ha permitido comprender mejor las necesidades de las usuarias y, así, crear mejores estrategias. Además, el riesgo de abandono de los procesos ha disminuido, ya que las usuarias manifiestan contar con espacios seguros que les permiten continuar, hasta el final, los procesos. En el caso de las personas estudiantes de derecho, estas han adquirido herramientas para realizar entrevistas a usuarias, desde una perspectiva más empática, lo que mejoró la calidad de los servicios ofrecidos (Mónica Sancho, comunicación personal, 1 de diciembre del 2017).

\section{Resultados generados a partir de un Trabajo Final de Graduación bajo la Modalidad de Práctica Dirigida}

Entre agosto del 2019 y marzo del 2020, se realizó una Práctica Dirigida para optar por el grado de Licenciatura en Psicología, titulado "Fortalecimiento del servicio de atención a mujeres afectadas por Violencia Ejercida en Relaciones de Pareja, que ofrece el Consultorio 
Jurídico de la Universidad de Costa Rica, Defensoría de los Habitantes, desde un enfoque género sensitivo y de derechos humanos" (Artavia 2021).

En este proceso, uno de sus ejes de trabajo consistió en crear procesos de atención que permitieran, a usuarias afectadas por la VERP, desarrollar estrategias para protegerse de la violencia. También, se generaron procesos de intervención en crisis, mediante el trabajo interdisciplinario y la generación de espacios seguros de atención, esto con el fin de disminuir situaciones de riesgo y aumentar los factores protectores contra la violencia.

A nivel de metodología, se utilizaron los aportes de Claramunt (1999), anteriormente mencionados, como marco de acción para brindar intervenciones en crisis acopladas a las necesidades las usuarias. Asimismo, cada una de las personas participantes contó con un consentimiento informado donde se explicaron todas las características del proceso de intervención (Artavia 2021).

En la misma línea, durante el proceso se realizaron sesiones individuales de intervención en crisis. Asimismo, en ocasiones se trabajó de forma conjunta con el estudiantado de derecho, para así brindar un acompañamiento interdisciplinario.

Durante esta experiencia, el trabajo psicológico consistió en realizar valoraciones de riesgo, de forma constante, en las que se identificaron factores que podrían vulnerabilizar a las usuarias y exponerlas a situaciones de riesgo; como el uso de armas de fuego por parte del agresor, la presencia de menores de edad y otros elementos contemplados por el Consejo Superior del Poder Judicial (2007). A partir de esta información, desde una perspectiva legal, se diseñaron estrategias para proteger a las usuarias, al mismo tiempo que, desde la Psicología, se buscó brindarles contención emocional, desculpabilizarlas y crear planes de seguridad para protegerlas, por medio de la identificación de redes de apoyo, así como estrategias para protegerse en caso de un episodio de violencia (Artavia 2021).

Aunado a lo anterior, se asesoró continuamente al equipo de Derecho, con el fin de que desarrollaran habilidades para generar espacios seguros de atención, por medio de estrategias para abordar a las usuarias, a través de la empatía y el respeto por sus decisiones. Asimismo, lograron identificar las necesidades de las víctimas para así contextualizar los servicios (Artavia 2021).

En relación con los resultados, la valoración de riesgo de femicidio demostró que las mujeres fueron violentadas principalmente por sus parejas, por medio de violencia 
psicológica y patrimonial, mayoritariamente. Las usuarias en mayor riesgo presentaron, también, violencia física y sexual. Producto de esto, sufrieron afectaciones a nivel físico, como, por ejemplo: abortos, enfermedades de transmisión sexual, fibromialgia y lesiones; mientras que, a nivel psicológico, algunas presentaron ideaciones suicidas años anteriores, depresión, ansiedad y miedo constante al estar expuestas a dichas situaciones de violencia (Artavia 2021).

A nivel de la ruta crítica, las usuarias reportaron vivir contradicciones porque no siempre los procesos legales eran como ellas esperaban, al ser muy largos o solicitar muchos requisitos. Además, fue evidente que, para ellas, aceptar su vivencia ante otras personas fue una etapa realmente difícil de asimilar, ya que les generó miedo y culpa (Artavia 2021).

En relación con las sesiones de intervención, se realizaron 58 espacios con 21 usuarias, donde 13 fueron procesos de intervención con mujeres afectadas por la VERP. Durante esta experiencia se logró demostrar que las mujeres recibieron acompañamiento en las diferentes etapas de los procesos legales, pero también mejoraron sus habilidades para regular sus emociones y cumplieron con metas establecidas durante la intervención. Asimismo, la mayoría de las usuarias aprendieron a desarrollar planes de seguridad y a reconectar con sus redes de apoyo, lo cual les permitió aumentar los factores protectores frente a la violencia. Además, acudieron a grupos de apoyo, terapia psicológica y desarrollaron planes para independizarse económicamente de sus excompañeros sentimentales. Cada sesión promovió el respeto de sus derechos humanos, lo que permitió que tuvieran la oportunidad de expresarse en un contexto seguro. (Artavia 2021).

A nivel de estrategias de protección, se les brindó información para comprender los factores de riesgo, se crearon espacios de empatía y reconocimiento emocional, así como se realizaron referencias a instituciones estatales que podrían brindar procesos de atención y acompañamiento para atender las necesidades y consecuencias generadas por la violencia (Artavia 2021).

De esta manera, al concluir el proceso se disminuyó el riesgo de femicidio de las usuarias, debido al desarrollo de nuevas herramientas para protegerse de la violencia. Para comprender mejor este impacto, la figura 1 muestra la valoración de riesgo realizada a 13 usuarias antes de iniciar el proceso y la figura 2 muestra el análisis posterior a las sesiones de acompañamiento e intervención en crisis: 


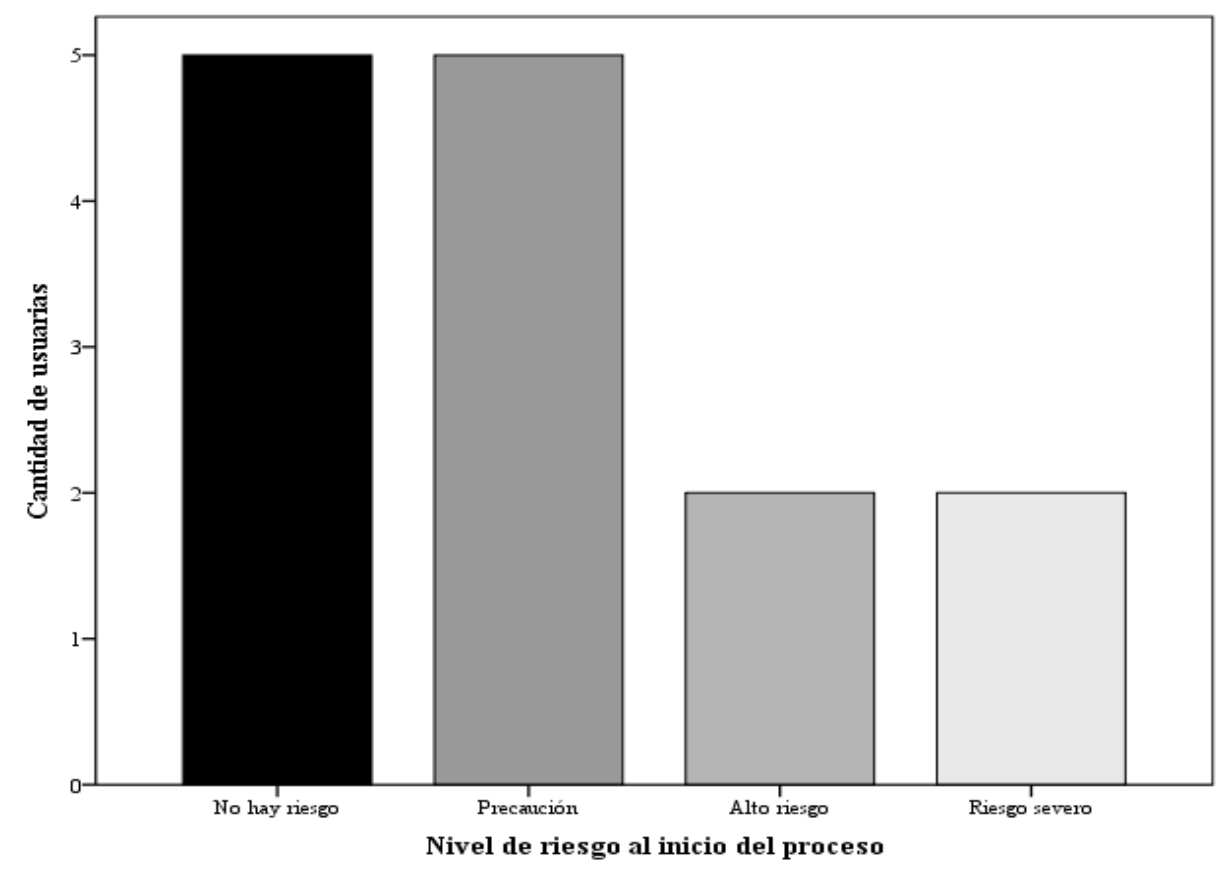

Figura 1. Nivel de riesgo de femicidio identificado en la vida de las usuarias al inicio del proceso de atención individual. Fuente: Artavia (2021 p. 52) 


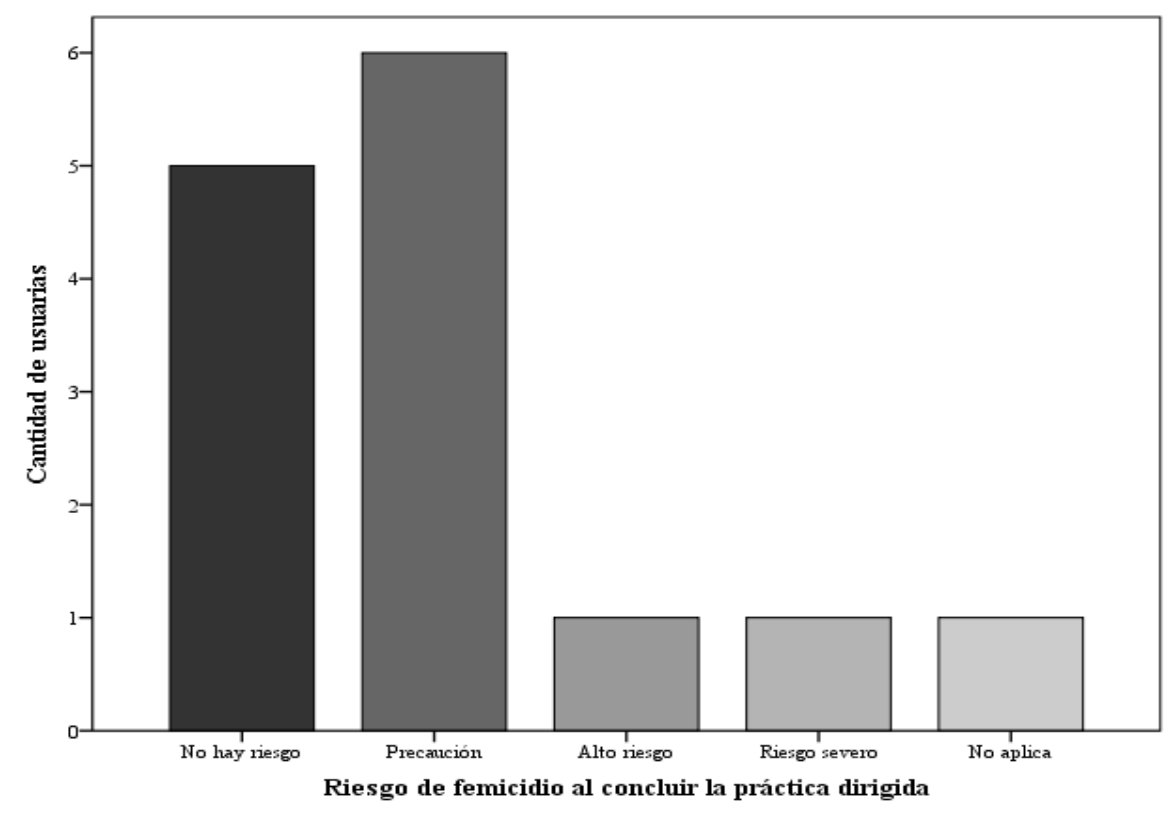

Figura 2. Nivel de riesgo de femicidio reportado por las usuarias al finalizar la práctica dirigida. Fuente: Artavia (2021 p.52).

A partir de lo anterior, se identifica que las usuarias lograron disminuir el riesgo de femicidio. Asimismo, al finalizar el proceso, las mujeres que se mantenían en alto riesgo o riesgo severo contaron con medidas de protección y planes de seguridad realizados a lo largo de la intervención, para así, poder protegerse. De igual forma, todas contaron con información sobre instituciones que podrían protegerlas en caso de riesgo, inclusive aquellas en estado de precaución donde lo que dominó fue la violencia psicológica. Es importante destacar que una usuaria abandonó el proceso luego de la primera sesión, por este motivo en la figura 2 se muestra que la valoración no se realizó con una participante (Artavia 2021).

Al finalizar este eje de atención individual, las mujeres atendidas comentaron que el espacio seguro les permitió comprender la importancia de cuidarse, protegerse y, sobre todo, reconocer el amor propio (Artavia 2021).

De esta manera, a partir de esta experiencia, fue evidente que el trabajo interdisciplinario, la identificación de situaciones de riesgo, así como el acompañamiento psicológico, por medio de la intervención en crisis, permitió a usuarias afectadas por la VERP, disminuir factores de riesgo y aumentar los protectores, al contar con herramientas 
legales para protegerse, pero, también, al contar con espacios seguros para poder expresarse, lo que estabilizó sus emociones y adquirir herramientas al no ser revictimizadas (Artavia 2021).

\section{Análisis y conclusiones}

De acuerdo a la experiencia desarrollada por Artavia (2021), es evidente que las usuarias del CJDHR vivieron relaciones desiguales de poder, en las que sus excompañeros sentimentales utilizaron la violencia para controlarlas y dominarlas (Abrahams 2010; Castro 2015; Sagot, Carcedo y Guido 2000; Instituto Nacional de las Mujeres 2016; Ulate 2008).

Por su parte, durante la intervención, las usuarias buscaron ayuda para acceder a la justicia, con el fin de proteger sus derechos humanos, lo que, en conjunto con la intervención interdisciplinaria, logró no solo fortalecer sus factores protectores, sino también contrarrestar efectos de la violencia tales como el aislamiento, por medio de la reconexión con sus principales redes de apoyo familiar (Abrahams 2010).

En cuanto a las formas de violencia, las usuarias demostraron que las distintas manifestaciones y consecuencias, efectivamente, son producidas por las conductas de sus compañeros sentimentales, lo que demostró que vivir continuamente en esta dinámica puede producir consecuencias a corto y largo plazo, que podrían atentar contra su supervivencia (Castro 2015; FRA 2015; Mahapatro 2018; Walker 2009). De esta manera, los resultados confirman las postulaciones de Abrahams (2010), Castro (2015) FRA (2015), el Instituto Nacional de las Mujeres (2016) y Ulate (2008) al afirmar que, las consecuencias de la VERP pueden afectar la calidad de vida de las mujeres.

Por otro lado, con relación a la ruta crítica, es evidente que se cumplen los postulados de Jiménez et al. (2013), ya que, para las víctimas, el comunicar su vivencia y buscar ayuda es un momento realmente difícil. Asimismo, si se compara esta información con los aportes de Claramunt (1999), Raffo (2007), Roberts (2005) y Yeager y Roberts (2015) se logra identificar el valor del acompañamiento psicológico con esta población, porque permite crear estrategias para proteger a las usuarias, pero también promover espacios de escucha, validar 
sus experiencias y, sobre todo, identificar situaciones de riesgo que podrían enfrentarse a través de procesos legales.

Aunado a lo anterior, al brindar apoyo a profesionales en Derecho, se logró comprender los procesos e identificar las necesidades de las usuarias, lo que permitió generar empatía. De esta manera, como lo explican Raffo (2007) y Claramunt (1999), el trabajo interdisciplinario y la intervención en crisis son vitales para comprender el contexto de las usuarias y brindar contención emocional al activar la ruta crítica, porque permiten crear espacios seguros. No obstante, es a través de las leyes y el reconocimiento de sus derechos humanos que se logran reducir, aún más, los factores de riesgo de femicidio, por lo cual, el abordaje individual de ambas disciplinas es insuficiente. Por este motivo, se considera fundamental la presencia del acompañamiento psicológico, para guiar al personal de derecho en las estrategias necesarias para generar prácticas más humanitarias (Raffo, 2007).

Finalmente, es evidente que las usuarias lograron aprender de sus experiencias de crisis, al adquirir conocimientos de ambas disciplinas sobre estrategias para protegerse de la violencia, por medio de un espacio de escucha, planes de seguridad y contención emocional, pero también a través de insumos legales que podrían protegerlas en situaciones de riesgo (Claramunt 1999; Raffo 2007; Roberts 2005; Yeager y Roberts 2015).

\section{Referencias Bibliográficas}

Adina Castro, ed., La Violencia Doméstica. Tenemos derecho a una vida sin violencia. (Costa Rica: INAMU, 2015).

Ana Rodríguez (2001). "Los Consultorios Jurídicos de la Universidad de Costa Rica: "Evolución Normativo-Social, Implicaciones de la Nueva Legislación en materia de familia, Situación Actual y Propuesta de Reestructuración” (Tesis de Licenciatura en Derecho, Universidad de Costa Rica, 2001).

Carmen Ulate. Violencia de género y violencia intrafamiliar: Módulo III. (Costa Rica: Instituto de Estudios de la Mujer, 2008), https://repositorio.una.ac.cr/bitstream/handle/11056/2464/recurso_562.pdf?sequenc $\mathrm{e}=1$ \&is Allowed=y 
Cecilia Claramunt, ed., Mujeres maltratadas. Guía de trabajo para la intervención en crisis. (Costa Rica: Organización Panamericana de la Salud, 1999).

Claudia Cervantes y Alonso Veraza. Documentación de la experiencia en la implementación de los Centros de Justicia para las Mujeres. (México: Banco Interamericano de Desarrollo, 2018), https://publications.iadb.org/bitstream/handle/11319/8913/Documentacion-de-laexperiencia-en-la-implementacion-de-los-Centros-de-Justicia-para-lasMujeres.pdf?sequence $=1 \&$ is Allowed $=\mathrm{y}$

Consejo Superior del Poder Judicial. 2007. "Protocolo interinstitucional intervención para la atención de mujeres en situación de riesgo alto de muerte por violencia”. Costa Rica.

Defensoría de los Habitantes. 2010. "Convenio de funcionamiento del Consultorio Jurídico de la Universidad de Costa Rica ubicado en las instalaciones de la Sede Central de la Defensoría de los Habitantes." Costa Rica.

European Union Agency for Fundamental Rights. Violence against women: an EU-wide survey (Unión Europea: Publications Office of the European Union, 2015).

Facultad de Derecho. 2014. "Manual Informativo y Didáctico del Área de Acción Social”. Costa Rica.

Hilary Abrahams, ed., Rebuilding Lives after Domestic Violence. (Reino Unido: Jessica Kingsley Publishers, 2010).

Instituto Nacional de las Mujeres. 2016. "Informe de rendición de cuentas. Período 20122013 “Avanzando de jure a de facto”. Área Violencia de Género, Instituto Nacional de las Mujeres". Costa Rica.

Kenneth Yeager y Albert Roberts, ed., Crisis intervention Handbook, Assessment, Treatment, and Research ( $3^{\mathrm{a}}$ ed.) (Inglaterra: Oxford University Press, 2015).

Leonore Walker, ed., The Battered Woman Syndrome (3 ${ }^{\mathrm{a}}$ ed.). (Estados Unidos: Springer Publishing Company, LLC., 2009). 
Ley de Consultorios. 1971. "Ley N 4775 del 21 junio de 1971.” Costa Rica

Marta Alvarado, Arlette Esquivel, Sonia Álvarez. "Análisis sobre la Asistencia Legal y Consultorios Jurídicos en Costa Rica. Ideas para una nueva etapa” (Seminario de Graduación de Licenciatura en Derecho, Universidad de Costa Rica, 1982).

Marta Artavia. "Fortalecimiento del servicio de atención a mujeres afectadas por Violencia Ejercida en Relaciones de Pareja, que ofrece el Consultorio Jurídico de la Universidad de Costa Rica, Defensoría de los Habitantes, desde un enfoque género sensitivo y de derechos humanos" (Práctica Dirigida de Licenciatura en Psicología, Universidad de Costa Rica, 2021).

Meerambika Mahapatro, ed., Domestic Violence and Health Care in India Policy and Practice. (India: Springer, 2018).

Monserrat Sagot, Ana Carcedo, Lea Guido, ed., La ruta crítica de las mujeres afectadas por la violencia intrafamiliar en América Latina (Estudios de Casos en 10 países). (América Latina: Zeta Servicios Gráficos, 2000).

Monserrat Sagot y Laura Guzmán. Informe Final de Investigación. (Costa Rica: Universidad de Costa $\quad$ Rica, 2004), http://www.colectivacr.com/sites/default/files/Docs/mat_int/Encuesta\%20Nacional\%20de\%20Violencia. pdf

Morelba Jiménez, Jessie Blanco, Susana Medina y Rosalba Gómez. Ruta Crítica de las mujeres afectadas por violencia de pareja en Venezuela (Venezuela: Fundación CBD Publicaciones, 2013).

Observatorio de Violencia de Género contra las Mujeres y acceso a la Justicia. "Violencia Doméstica”. Acceso el 26 de Junio del 2020, https://observatoriodegenero.poderjudicial.go.cr/soy-especialista-y-busco/estadisticas/violencia-domestica/

Observatorio de Violencia de Género contra las Mujeres y acceso a la Justicia. "Femicidio" Acceso el 8 de noviembre del 2020, https://observatoriodegenero.poderjudicial.go.cr/index.php/soy-especialista-y-busco/estadisticas/femicidio 


\section{वrivum}

Wimblu, Rev. Estud. de Psicología UCR, 16(2) 2021 (Julio-Diciembre): 119-136 /ISSN: 1659-2107

Organización de Estados Americanos. 1994. "Convención Interamericana Para Prevenir, Sancionar Y Erradicar La Violencia Contra La Mujer (Convención De Belém Do Pará).". Brasil.

Organización Mundial de la Salud. "Violencia Contra La Mujer". Acceso el 29 de noviembre del 2017, https://www.who.int/es/news-room/fact-sheets/detail/violence-againstwomen.

Pilar Raffo, ed., Atención integral a víctimas de tortura en procesos de litigio. (Costa Rica: Instituto Interamericano de Derechos Humanos, 2007).

Albert Roberts, ed., Crisis Intervention Handbook: Assessment, Treatment and Research. (Inglaterra: Oxford University Press, 2005). 